\title{
ANÁLISE DO DESEMPENHO ACÚSTICO DE FACHADAS VENTILADAS DE PORCELANATO À LUZ DA NORMA DE DESEMPENHO: ESTUDO DE CASO EM UM EDIFÍCIO HABITACIONAL EM BRASÍLIA-DF
}

\author{
Acoustic Performance Analysis of Porcelain Ventilated Façades \\ Considering the Performance Standard: Case Study for a Residential \\ Building in Brasília-DF
}

\author{
Vamberto Machado dos Santos Filho', Rosa Maria Sposto ${ }^{2}$, Lucas Rosse Caldas ${ }^{3}$
}

Recebido em 13 de janeiro de 2017; recebido para revisão em 06 de março de 2017; aceito em 08 de maio de 2017; disponivel on-line em 15 de maio de 2017.

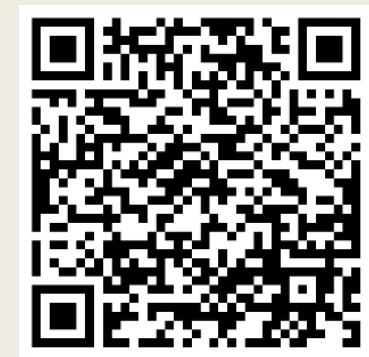

PALAVRAS CHAVE:

Fachadas ventiladas; Revestimento de porcelanato;

Desempenho das edificações; Simulação computacional.

KEYWORDS:

Ventilated façades; Porcelain tiles covering; Building performance; Computer simulation.

* Contato com os autores:

Doutorando do Programa de Pós-Graduação em Estruturas e Construção Civil da Universidade de Brasília - UnB.

2e-mail: rmsposto@unb.br (R. M. Sposto)

Professora, Doutora, Programa de Pós-Graduação em Estruturas e Construção Civil da Universidade de Brasília - UnB.

${ }^{3}$ e-mail: Irc.ambiental@gmail.com (L. R. Caldas)

Doutorando do Programa de Engenharia Civil - COPPE - Universidade Federal do Rio de Janeiro - UFRJ. 


\section{INTRODUÇÃO}

Atualmente é comum assistirmos, nos grandes centros urbanos, a substituição de fachadas convencionais, de alvenaria, revestimento cerâmico aderido ou pintura por fachadas pré-fabricadas, de concreto ou metálicas do tipo ventiladas, ou seja, com camada de ar entre o substrato e o revestimento externo. As fachadas ventiladas diferem das fachadas cortinas pela presença desta camada de ar renovado constantemente.

Apesar da comprovada melhoria no desempenho térmico deste tipo de fachada, pouco se sabe a respeito do seu desempenho acústico (WONG et al., 2008; STAZI et al., 2011; LOPÉZ et al., 2012; SUÁREZ et al., 2012; MARTINS, 2013; LOPÉZ et al., 2015; e SANTOS FILHO et al., 2015).

Gerretsen (2003) afirma que os elementos das construções estão se tornando cada vez mais leves para facilitar a pré-fabricação. As fachadas, as divisórias de ambientes e as lajes de cobertura são cada vez menos espessas, acarretando maior transmissão sonora (QUEIROZ, 2007).

Castro et al. (2015), OMS (2014), Nunes e Ribeiro (2008) e Pimentel-Souza (1997) indicam que o ruído é um dos principais causadores de distúrbios de sono, estresse e perturbação do ritmo biológico. Estes autores ainda apontam que a exposição ao ruído provoca, direta ou indiretamente, aumento do risco de enfarte, derrame cerebral, infecções, fadiga mental, além da falta de privacidade da habitação, que gera insatisfação, irritação e a violência no convívio social.

No Brasil, o desempenho acústico nas edificações foi sendo negligenciado durante muito tempo. Esse panorama das construções brasileiras é preocupante, já que o ruído urbano aumentou enquanto $O$ isolamento sonoro perdeu eficiência (DUARTE, 2005).

De acordo com Hammad (1983) em países de clima quente, fatores como a proteção térmica, insolação e ventilação são mais consideradas que os parâmetros acústicos.

Em relação às edificações é possível diminuir o ruído sonoro em seu interior adotando medidas de isolamento nos elementos de fachada.
Para isso é necessário saber qual a solicitação sonora do ambiente em estudo, para um correto dimensionamento desses elementos (dimensionamento utilizando previsão). Garavelli et al. (2010), Maciel et al. (2009), Nunes e Ribeiro (2008) avaliaram a incidência de ruídos intensos nas edificações localizadas em cidades do Distrito Federal, enquanto Ferreira e Zannin (2007), Queiroz (2007), Alcantara (2010), Asakura et al. (2013), Dintrans e Préndez (2013), utilizaram de softwares para prever níveis de ruído no interior de uma edificação.

Considerando a NBR 15.575 (ABNT, 2013) também conhecida como norma de desempenho, que visa o atendimento às exigências dos usuários, com base em requisitos e critérios de segurança, habitabilidade e sustentabilidade, há necessidade da verificação da fachada em relação ao desempenho acústico.

Neste contexto, o presente trabalho se justifica por avaliar o atendimento dos níveis de desempenho acústico de fachadas ventiladas de porcelanato (FVPo) e comparar esse sistema inovador com o sistema convencional de fachada de porcelanato aderido (FPoA).

\section{OBJETIVOS}

Esse trabalho tem como objetivo verificar o desempenho acústico de um Edifício Habitacional Modelo (EHM) composto por um sistema de fachada ventilada de porcelanato, em Brasília-DF, à luz dos requisitos e critérios da NBR 15.575 (ABNT, 2013).

\section{REVISÃO BIBLIOGRÁFICA}

\subsection{PROBLEMÁTICA DO RUÍDO URBANO}

De acordo com Corbella e Yannas (2003), o som pode ser caracterizado como uma sensação causada no ouvido por variações da pressão do ar, resultado de uma fonte de som, que pode ser móvel (cordas vocais, autofalantes) ou o ar em movimento (motores, ventiladores e etc.). Já o ruído pode ser definido, segundo Knudsen e Harris (1959), como um som indesejável. Desta forma o ruído se 
caracteriza pela existência de muitas amplitudes e frequências ocorrendo simultaneamente de maneira não harmônica. Calixto (2002) ressalta que a fronteira entre o som e o ruído não pode ser definida com precisão, pois cada indivíduo apresenta uma reação diferente ao som e ao ruído, que depende dentre outros fatores, de seu estado emocional e de sua personalidade.

No Brasil existem normas e legislação especifica para o controle do ruído urbano. A Resolução do Conselho Nacional do Meio Ambiente (CONAMA) $n^{\circ} 002$ (BRASIL, 1990) instituiu o Programa Nacional de Educação e Controle de Poluição - intitulado Silêncio, que considera problemática a poluição sonora nas áreas urbanas, indicando que o ruído em excesso é uma séria ameaça à saúde, ao bem-estar público e a qualidade de vida. Além de instituir o programa Silêncio, a resolução respeita os padrões estabelecidos pela NBR 10.151 (ABNT, 2000). Essa norma fixa valores máximos de comparação do nível de pressão sonora equivalente medido na banda $A$ (LAeq) com os valores de nível de critério de avaliação (NCA) por tipos de áreas e por períodos do dia (Tabela 1).

No Distrito Federal, a poluição sonora e seus problemas são abordados na Lei Distrital no 4.092 de Janeiro de 2008 (DISTRITO FEDERAL, 2008), que basicamente, segue o descrito na NBR 10.151 (ABNT, 2000).

De acordo com Queiroz (2007) o som e eventuais ruídos se propagam por meio de ondas em qualquer meio elástico. Essas ondas incidem sobre uma superfície (fachadas, divisórias internas, cobertura...) resultando na vibração destes. Neste processo, que ocorre a transferência de energia, parte dela é transmitida, parte é absorvida e parte é refletida. Portanto, a atenuação da propagação de ondas sonoras é imprescindível em uma edificação.

\subsection{DESEMPENHO ACÚSTICO NAS EDIFICAÇÕES}

De acordo com CBIC (2013), existem dois tipos de ruídos que são avaliados nas edificações: o ruído aéreo, definido como aquele que se propaga pelo ar e o ruído de impacto, aquele que apresenta picos de energia acústica de duração inferior a um segundo. Corbella e Yannas (2003) defendem que um bom projeto, do ponto de vista da acústica tem os seguintes objetivos: exclusão ou amortecimento do ruído externo e redução do som que passa de um ambiente para o outro.

Segundo a NBR 15.575-1 (ABNT, 2013) as edificações habitacionais devem apresentar isolamento acústico adequado das vedações verticais e coberturas, relacionado aos ruídos aéreos provenientes do ambiente exterior, como também isolamento acústico adequado entre as áreas comuns, privativas e unidades autônomas. Referente às vedações verticais, é necessário o atendimento de dois critérios para um desempenho acústico satisfatório: índice de redução sonora ponderado (Rw) e diferença padronizada de nível ponderada a $2 \mathrm{~m}$ de distância de fachada $(\mathrm{D} 2 \mathrm{~m}, \mathrm{nT}, \mathrm{w}))$, sendo o primeiro obtido em laboratório e o segundo medido em campo. Os valores medidos em campo tendem a ser menores que os obtidos em laboratório.

TABELA 1: Nível de critério de avaliação NCA para ambientes externos, em dB(A).

\begin{tabular}{c|c|c} 
Tipos de áreas & Diurno & Noturno \\
\hline Áreas de sítios e fazendas & 40 & 35 \\
\hline Áreas estritamente residencial urbana ou de hospitais ou de escolas & 50 & 45 \\
\hline Área mista, predominantemente residencial & 55 & 50 \\
\hline Área mista, com vocação comercial e administrativa & 60 & 55 \\
\hline Área mista, com vocação recreacional & 65 & 60 \\
\hline Área predominantemente industrial & 70 & 65 \\
\hline
\end{tabular}

FONTE: NBR 10.151 (ABNT, 2000). 
Desta forma, torna-se necessário a proteção dos usuários da edificação, por meio do isolamento acústico de sua envoltória, projetando dentre os diversos elementos da fachada (janelas, paredes, revestimentos) redutores sonoros. É possível estimar o desempenho do isolamento da edificação através da avaliação do desempenho de cada elemento, de acordo com a norma ISO 157123: 2005 (SANTOS FILHO, 2015).

Os níveis de desempenho mínimos, intermediários e superiores para o índice Rw, de acordo com a classe de ruído do meio, podem ser verificados na Tabela 2.

Ferreira e Zannin (2007) avaliaram o desempenho acústico de habitações em alvenaria localizadas em Curitiba por meio da medição in situ e simulação computacional. Primeiramente, por meio da análise estatística concluíram a concordância dos resultados encontrados nas duas formas de medição. Por fim, os resultados obtidos, o índice de isolação sonora entre os cômodos e aparente das fachadas, apresentaram valores inferiores aos previstos nas normas internacionais.

Já Garcia et al. (2008), avaliaram o desempenho de isolamento sonoro aéreo por meio de expressões analíticas e experimentais para diferentes tipologias de painéis de vedação vertical, classificados pelo autor como painéis maciços, duplos e sanduíche. Os autores concluíram que as diferenças entre os resultados dos procedimentos analíticos e experimentais demonstraram certa dificuldade em predizer o isolamento dos componentes analisados por meio de equações. Os painéis maciços, compostos de mais camadas, não foram bem representados pelas equações empregadas, enquanto para os outros tipos de painéis houve uma boa concordância entre as equações e o ensaio experimental.

\subsection{FACHADAS VENTILADAS}

Segundo Kiss (1999) achada ventilada é um sistema de revestimento externo caracterizado pela existência de uma camada isolante sobre a vedação e uma camada externa de revestimento, composta de painéis modulares, fixada na edificação por uma estrutura metálica. O sistema deve prever um espaço vazio que permita, por efeito chaminé, uma ventilação contínua.

As fachadas ventiladas são largamente empregadas em países europeus por apresentarem características que beneficiam questões de eficiência energética, estética, resistência, potencial criativo e conforto ambiental das edificações, o que o faz um sistema competitivo, especialmente para retrofit e restauro de edificações (CAMPOS, 2011).

Estas fachadas apresentam algumas vantagens quando comparadas ao sistema convencional, entre elas: maior isolamento térmico como resultado do fluxo de ar entre as camadas; diminuição dos efeitos de dilatação térmica na estrutura e consequente redução das manifestações patológicas associadas a este efeito; redução do efeito de condensação no seu interior; melhoria no isolamento acústico como resultado da câmara de ar; facilidade de manutenção e substituição; montagem industrializada e racional; e maior produtividade (CUNHA, 2006).

TABELA 2: Índice de redução sonora ponderado, Rw, de fachadas.

\begin{tabular}{|c|c|c|c|}
\hline $\begin{array}{l}\text { Classe de } \\
\text { ruído }\end{array}$ & Localização da habitação & $\operatorname{Rw}(\mathrm{dB})$ & $\begin{array}{c}\text { Nível de } \\
\text { desempenho }\end{array}$ \\
\hline \multirow{3}{*}{ I } & \multirow{3}{*}{$\begin{array}{l}\text { Habitação localizada distante de fontes de ruído intenso de } \\
\text { quaisquer naturezas }\end{array}$} & $\geq 25$ & $\mathrm{M}$ \\
\hline & & $\geq 30$ & 1 \\
\hline & & $\geq 35$ & $\mathrm{~S}$ \\
\hline \multirow{3}{*}{ II } & \multirow{3}{*}{$\begin{array}{l}\text { Habitação localizada em áreas sujeitas a situações de ruído não } \\
\text { enquadráveis nas classes I e III }\end{array}$} & $\geq 30$ & $\mathrm{M}$ \\
\hline & & $\geq 35$ & 1 \\
\hline & & $\geq 40$ & $S$ \\
\hline \multirow{3}{*}{ III } & \multirow{3}{*}{$\begin{array}{l}\text { Habitação sujeita a ruído intenso de meios de transporte e de } \\
\text { outras naturezas, desde que conforme a legislação }\end{array}$} & $\geq 35$ & $\mathrm{M}$ \\
\hline & & $\geq 40$ & 1 \\
\hline & & $\geq 45$ & $\mathrm{~S}$ \\
\hline
\end{tabular}


Apesar das vantagens, o sistema possui algumas limitações de uso, podendo ser associados à ausência de normas específicas, a necessidade de mão de obra qualificada, a exigência de projetos mais complexos, e o alto preço relativo (SANTOS FILHO, 2015)

Ainda segundo o mesmo autor, no Brasil, as fachadas ventiladas, em sua grande maioria, são comercializadas em forma de sistema. As empresas especializadas vendem toda a concepção do sistema, desde a análise do empreendimento, a elaboração do projeto de fachadas, a consultoria, a especificação e a quantificação dos materiais, incluindo a montagem. Os revestimentos externos empregados variam, podendo ser de porcelanato, placas pétreas ou alumínio composto (ACM).

Estudos relacionados às fachadas ventiladas em sua grande maioria tem se voltado para questões relacionadas ao desempenho térmico e de eficiência energética

Muller (2003) comparou o desempenho térmico de uma fachada ventilada de placas cerâmicas de porcelanato com fachada convencional para as condições de verão e inverno do Sul do Brasil.

Sanjuan et al. (2011) compararam o desempenho energético entre uma fachada ventilada e uma convencional e concluíram o melhor desempenho da fachada ventilada.

Aparicio-Fernández et al. (2014) avaliaram o desempenho térmico e a consequência no desempenho energético de uma edificação de escritórios localizada em Valência na Espanha. Os resultados deste estudo mostraram a redução no consumo de energia devido ao aquecimento.

No entanto, relacionado ao desempenho acústico, não se tem ainda dados suficientes para se afirmar sobre o desempenho das fachadas ventiladas, que é o questionamento deste trabalho.

\section{METODOLOGIA}

A metodologia deste trabalho está dividida em três etapas principais:

1. Caracterização do sistema: Definição das variáveis que influenciam na análise do sistema proposto, levando em consideração características físicas e geográficas da edificação e das vias estudadas.

2. Simulação computacional: Análise numérica computacional utilizando software de desempenho acústico.

3. Análise e Resultados: Comparação dos resultados obtidos das simulações e sua análise.

\subsection{CARACTERIZAÇÃO DO SISTEMA}

Para a realização desse estudo utilizou-se um projeto de sistema de vedação vertical externa SVVE de fachada ventilada típica do plano piloto elaborado por Maciel (2013), a partir de observação das principais tipologias e materiais do plano piloto do DF, considerando pilotis, 6 pavimentos e cobertura. As variáveis consideradas no estudo são o porcelanato com insert metálico (FVPo) e o porcelanato aderido ao substrato (FPoA), utilizada como referência.

O projeto é constituído por 03 (três) blocos germinados simétricos, sendo que cada bloco possui 04 (quatro) apartamentos por andar com 02 (dois) elevadores, escada de incêndio, hall social e depósito de lixo (Figura 1). Os apartamentos possuem 03 (três) quartos sendo uma suíte, banho social, sala de estar, cozinha, área de serviço e varanda.
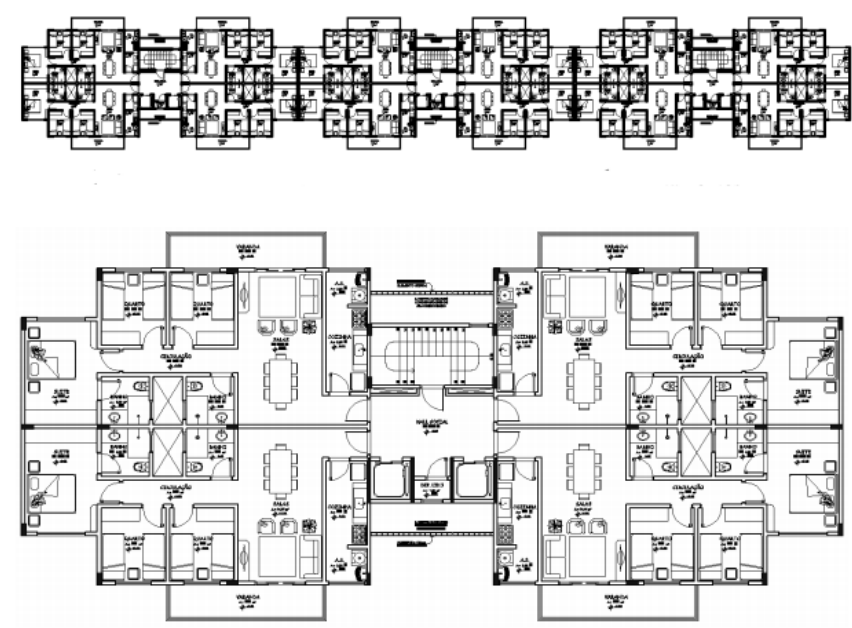

FIGURA 1 - Planta baixa do edifício e do bloco germinado (MACIEL, 2013).

O sistema construtivo considerado é estrutura de concreto armado com lajes de $10 \mathrm{~cm}$ de espessura (incluindo a laje de cobertura), pé 
direito de 2,90 metros, contra piso de $2 \mathrm{~cm}$, vedações verticais de bloco de concreto de dimensões de $14 \mathrm{~cm} \times 19 \mathrm{~cm} \times 39 \mathrm{~cm}$ com juntas verticais e horizontais de $1 \mathrm{~cm}$ de espessura com argamassa de cimento, areia e cal e revestimento interno com reboco paulista de $1,5 \mathrm{~cm}$ de espessura com argamassa de cimento, areia e cal.

\subsection{RUÍDO DE TRÁFEGO}

Para a obtenção dos valores de ruído de tráfego que serão considerados nas fachadas estudadas, foram necessárias 5 etapas: 1) Caracterização espacial do ambiente de estudo; 2) Obtenção dos dados de fluxo de tráfego das vias; 3) Cálculo dos níveis de predição sonora; 4) Localização dos locais de medição e 5) Medição in loco dos níveis de pressão sonora equivalentes com ponderação $A$ (LAeq).

Na primeira etapa, foram identificadas as principais fontes de ruídos que afetam as edificações no local escolhido para a análise. No caso, o eixo rodoviário oeste (eixo $W$ ) e o eixo rodoviário de Brasília (DF 002) são os principais produtores de ruídos sonoros, o que vai ao encontro das ideias de Mardones (2009), que aponta o fluxo de veículos como umas das principais fontes de ruído. Os efeitos da atenuação do ruído de tráfego pela vegetação foram desconsiderados, já que, como afirma Hendricks (1995), a faixa de vegetação (20 metros) não é suficiente para causar significativas reduções sonoras.
Posteriormente, foram obtidos os dados de fluxo veicular, analisando os relatórios cedidos pelos Departamentos de Estrada e Rodagem (DERDF) e o Departamento de Trânsito do Distrito Federal (DETRAN-DF). Os relatórios são realizados com o auxílio dos CEVs (Controlados eletrônicos de velocidade) que além de fotografar os veículos que ultrapassam o limite de velocidade da via, registram ao longo das 24 horas do dia, por hora, o número total de veículos que passam naquela via. Os relatórios fornecidos contêm informações do fluxo de veículos, por porte veicular, por hora, durante os meses de Abril e Maio de 2015,

Em posse dos relatórios de fluxo veicular nas vias a serem analisadas, foram identificados os horários de maior fluxo veicular. A identificação dos picos de fluxo veicular foi necessária para a escolha dos horários de medição, que no caso foi de 7 às 9 horas e das 17 às 19 horas.

Foram realizados os cálculos de predição do nível de pressão sonora de acordo com os modelos propostos por diversos autores (Tabela 3) e posteriormente comparados com os resultados medidos. As equações de predição de nível de pressão sonora de tráfego utilizam de diversas variáveis como, por exemplo, o fluxo veicular (q), fluxo de veículos leves (VL) e pesados (VP), velocidade médias (V) entres outros. Esses valores são de grande importância para a classificação da edificação nas classes de ruído da norma de desempenho.

TABELA 3: Equações de predição do nível de pressão sonora.

\begin{tabular}{c|c} 
Autor & Equação de predição do nível de pressão sonora \\
\hline HMSO (1988) & $\begin{array}{c}L_{10}=10 \log 10 q+33 \log 10\left(v+40+\frac{500}{v}\right)+10 \log 10\left(1+\frac{5 p}{v}\right)-26,6, \\
\text { onde } L_{10}=L_{e q}+3\end{array}$ \\
\hline Garcia e Faus (1991) & $L_{e q}=48,6+8,1 \log (q)$ \\
\hline Sattler et al. (1996) & $L_{A e q}=65,4+4,67 \cdot 10^{-3} \cdot(q)$ \\
\hline Sattler et al. (1996) & $L_{A e q}=38,6+10,97 \cdot \log (q)$ \\
\hline Chakrabarty (1997) & $L_{e q}+20 \log d=53,8+17,2 \log q$ \\
\hline Nunes et al. (1999) & $L_{A e q}=8,0176 \log (q)+51$ \\
\hline
\end{tabular}


Na quarta etapa, analisaram-se os dados apresentados pelo mapa de ruído elaborado pelo Instituto Brasília Ambiental (IBRAM, 2013), a fim de escolher os locais de medição. No relatório desenvolvido pelo IBRAM são apresentados os mapas de ruído das principais vias de Brasília, entre elas o eixo w e a DF-002.

Por fim, procedeu-se à realização das medições in loco dos níveis de pressão sonora relativos ao fluxo de veículos. Para essa medição, foi utilizado o equipamento Minipa MSL-1352C, ponderado na curva de calibração $A^{1}$, seguindo as diretrizes da NBR 10.151 (ABNT, 2000), que especifica o método de medição de ruído aéreo externo. 0 equipamento foi montado a 1,2 metros do chão e distante no mínimo de 2 metros de qualquer superfície refletora, conforme mostrado na Figura 2. As medições aconteceram durante 20 minutos no período compreendido entre ás 7-9 horas e das 17-19 horas, de segunda a sexta do mês de novembro, sendo definidos intervalos de registro de dados de 5 segundos, no nível de resposta rápida (fast) do equipamento, durante o período de medição.

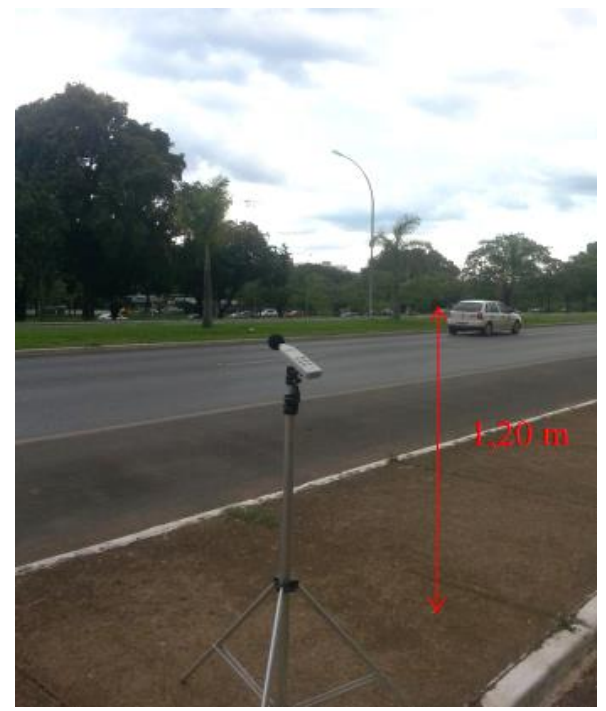

FIGURA 2: Montagem e posicionamento do equipamento de medição. FONTE: Autoria própria.

Após as leituras, calculou-se o nível de pressão sonora equivalente ponderado em $A$

${ }^{1}$ A curva de ponderação A é a mais utilizada nas medições acústicas por correlacionar os valores medidos com a incomodidade ou risco de trauma obtido. Essa curva atenua os sons graves, dando maior expressividade utilizando a equação da NBR 10.151 (ABNT, 2000) (Equação 1), em que: Li é o nível de pressão sonora lida em resposta rápida (fast) a cada 5 segundos, durante o período de medição do ruído e $\mathrm{n}$ é o número total de leitura.

$$
L_{\text {Aeq }}=10 \log \frac{1}{n} \sum_{i=1}^{n} 10^{\frac{L i}{10}}
$$

Finalmente, os dados obtidos pelos cálculos dos níveis de pressão sonora utilizando as equações da literatura, a verificação do mapa de ruídos de Brasília e a quantificação in loco, foram comparados e analisados quanto a sua representatividade.

\subsection{SIMULAÇÃO COMPUTACIONAL}

Para a simulação computacional foi utilizado o software Insul. Esse software tem como finalidade o cálculo de predição do isolamento sonoro de uma vedação. Esse cálculo é baseado em equações teóricas (teoria das massas, frequência crítica e outros) com o intuito de auxiliar projetistas na escolha dos melhores materiais para projetos de acústica.

Esse software possui algumas limitações, como por exemplo, a impossibilidade de cálculo de isolamento sonoro de todo o sistema de fachada, considerando todos os elementos simultaneamente. Para superar essa limitação, foi necessário simular os sistemas propostos para esse trabalho (FVPo e FPoA) separados das esquadrias, e posteriormente, calcular o índice de redução sonora aparente (RG) conforme a Equação 2.

$$
R_{G}=-10 \log \frac{\sum_{i}\left(S_{i} 10^{-\frac{R_{i}}{10}}\right)}{\sum S}
$$

Em que:

$R_{G}$ é o índice de redução sonora aparente da fachada;

$\mathrm{R}_{i}$ é o índice de redução sonora do elemento;

$S_{i}$ é a área do elemento da fachada; $\mathrm{e}$ S é a área total da fachada.

para a banda entre 2 a $5 \mathrm{KHz}$, voltando a atenuar sons mais agudos, aproximando assim, da percepção do som do ouvido humano. 


\subsubsection{Ruído incidente}

O início da simulação se dá pela inserção dos dados referentes ao tipo de ruído que o elemento a ser simulado está solicitado. Como explicado em 3.2, o ruído predominante no local de estudo é o de tráfego, e sua intensidade foi obtida conforme a Equação 1. Ao inserir os dados de entrada de intensidade sonora o software gera um gráfico com as faixas de frequência e intensidade típica de ruídos de tráfego (Figura 3), calculados conforme ISO 717-1 (2013).

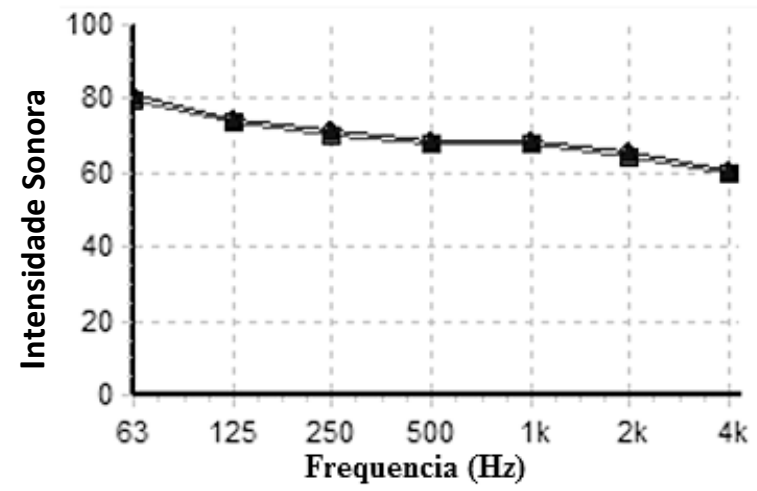

FIGURA 3 - Faixas de frequências do ruído de tráfego estudado.

FONTE: Autoria própria.

\subsubsection{Materiais utilizados}

Os materiais utilizados seguiram as especificações dos sistemas estudados (FVPo e
FPoA) e as suas características e esquemas de simulação estão descritos na Tabela 4. Para o cálculo do isolamento sonoro, adotou-se a contribuição da camada de ar e das juntas entre as placas de porcelanato do sistema de FVPo, como zero.

No estudo do isolamento sonora da fachada foram considerados 3 ambientes na edificação: 0 quarto 1 , a suíte e a sala (ambientes com permanência prolongada) e verificada a sua adequação conforme a norma. Na Tabela 5 é possível verificar as especificações dos ambientes estudados.

Na construção dos modelos de simulação utilizou-se a incidência de ondas na superfície da vedação de forma aleatória. No sistema de FPoA foi simulado, primeiramente, a vedação e depois a esquadria. Já para o sistema de FVPo, devido as limitações do software, foi necessário a simulação do revestimento de porcelanato acrescido de suas juntas e, posteriormente, a simulação do restante do sistema. A soma entre essas duas etapas foi realizada em escala logarítmica.

De acordo com a ISO 15712:2005, a transmissão sonora em fachadas acontece pela transmissão sonora de cada elemento constituinte da fachada, podendo assim ser calculado o índice de redução sonora da fachada (RG), ponderando as reduções sonoras de cada um de seus elementos.

TABELA 4: Características físicas dos materiais utilizados inseridas no software.

\begin{tabular}{c|c|c|c|c} 
Material & $\begin{array}{c}\text { Módulo de } \\
\text { elasticidade }(\mathbf{G P a})\end{array}$ & $\begin{array}{c}\text { Densidade } \\
\left(\mathbf{K g} / \mathbf{m}^{\mathbf{3}}\right)\end{array}$ & $\begin{array}{c}\text { Coeficiente } \\
\text { de Poisson }\end{array}$ & Fonte \\
\hline Argamassa & 4,1 & 1850,0 & 0,1 & Bastos et al. (2010) \\
\hline Bloco de concreto & 6,22 & 1880,0 & 0,3 & Juste (2001) \\
\hline Porcelanato & 69 & 2600,0 & 0,26 & Stubna et al. (1992) \\
\hline Vidro & 52,2 & 2430,0 & 0,3 & Makishima et al. (1975) \\
\hline
\end{tabular}

FONTE: Autoria própria.

TABELA 5: Características dos ambientes estudados.

\begin{tabular}{c|c|c|c} 
Ambiente & Área interna $\left(\mathbf{m}^{\mathbf{2}}\right)$ & Área da fachada $\left(\mathbf{m}^{\mathbf{2}}\right)$ & Área da esquadria $\left(\mathbf{m}^{\mathbf{2}}\right)$ \\
\hline Suíte & 24,40 & 9,17 & 2,40 \\
\hline Quarto & 8,24 & 8,51 & 1,80 \\
\hline Sala & 13,80 & 10,19 & 4,41 \\
\hline
\end{tabular}

FONTE: Autoria própria. 
Essa ponderação acontece através do valor das reduções sonoras (Rw) e de suas respectivas áreas no plano da fachada observada, conforme a Equação 2, apresentada anteriormente.

\section{RESULTADOS E DISCUSSÕES}

\subsection{NÍVEL DE PREDIÇÃO SONOERA E CLASSE DE RUÍDO}

Como resultado da aplicação dos modelos de predição do nível sonoro é possível verificar a diferença gerada entre cada uma delas. Na Figura 4 encontra-se o comportamento de cada equação mediante ao fluxo de veículos das vias estudadas.

É possível verificar que em alguns momentos as equações convergem para um valor próximo, geralmente em momentos de baixo fluxo de carros (entre 5 e 7 horas), onde o ruído de fundo é predominante na medição. Isso acontece quando o fluxo de tráfego é tão pequeno que, estatisticamente, o ruído produzido pelo tráfego não é expressivo, preponderando à medição do ruído de fundo.

Já no restante das horas, as equações se distanciam, como por exemplo, a de Charkrabarty (1997), que extrapola as outras equações e mostra um nível sonoro muito maior que os demais. Isso provavelmente acontece devido à peculiaridade dos dados obtidos por este autor, onde as suas medições aconteceram em Calcutá, além de ter sido dado enfoque nos usuários das edificações.

Em relação à correlação entre os valores obtidos pelas equações e os valores medidos in loco houve algumas diferenças. Devido ao urbanismo único de Brasília, os edifícios encontram-se afastados das vias de grande fluxo em média por 20 metros, diminuindo assim, a parcela de intensidade sonora refletida pelos mesmos. As equações também não levam em consideração a diminuição do fluxo de veículos e da velocidade, devido a congestionamentos, comuns nessas vias analisadas. Pode-se concluir que Brasília devido a suas peculiaridades necessita de uma equação de predição própria.

Na Tabela 6 é possível observar a correlação entre as equações de predição e os níveis medidos.

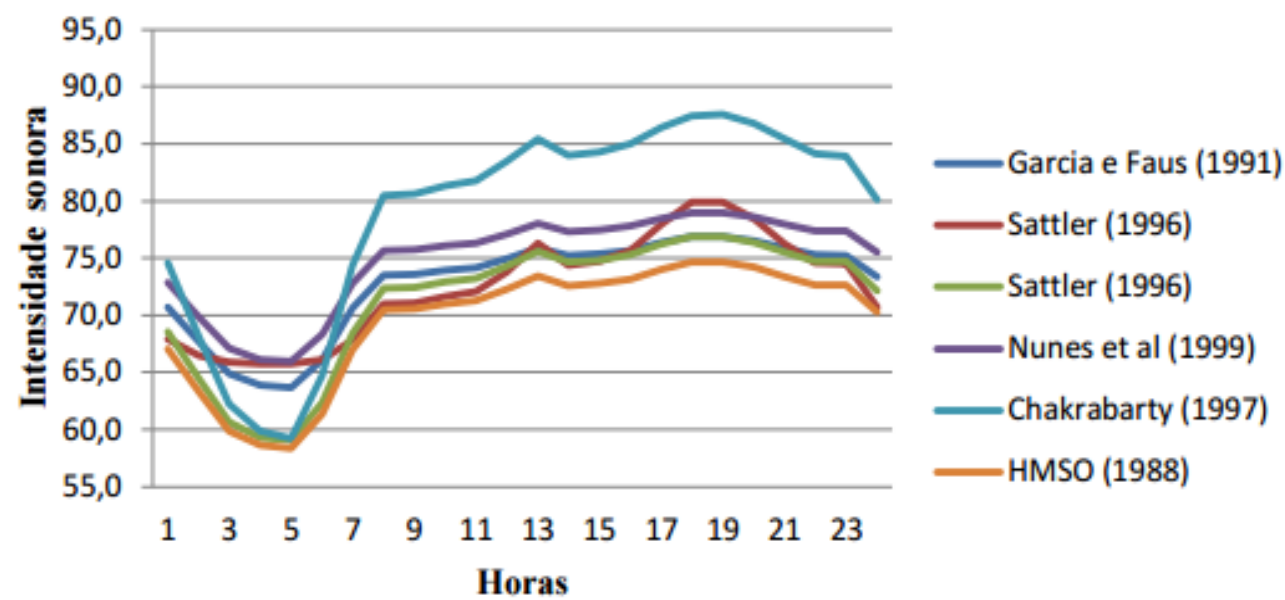

FIGURA 4 - Comportamento das equações de predição sonora. Fonte: Autoria própria. 
TABELA 6: Valores obtidos das equações de predição e medição in loco (dB).

\begin{tabular}{c|c|c|c|c|c|c|c} 
Hora & $\begin{array}{c}\text { Garcia e Faus } \\
\text { (1991) }\end{array}$ & $\begin{array}{c}\text { Sattler } \\
\mathbf{( 1 9 9 8 ) ~ L ~}\end{array}$ & $\begin{array}{c}\text { Sattler (1996) } \\
\text { NL }\end{array}$ & $\begin{array}{c}\text { Nunes et } \\
\text { al. (1999) }\end{array}$ & $\begin{array}{c}\text { Chakrabarty } \\
\text { (1997) }\end{array}$ & $\begin{array}{c}\text { HMSO } \\
\text { (1988) }\end{array}$ & Medido \\
\hline 7 & 73,5 & 71,0 & 72,4 & 75,7 & 80,4 & 70,5 & 69,4 \\
\hline 8 & 73,6 & 71,1 & 72,4 & 75,7 & 80,6 & 70,6 & 70,3 \\
\hline 9 & 73,9 & 71,7 & 72,9 & 76,1 & 81,3 & 71,0 & 72,5 \\
\hline 17 & 76,9 & 79,9 & 76,9 & 79,0 & 87,4 & 74,7 & 72,1 \\
\hline 18 & 76,9 & 79,9 & 76,9 & 79,0 & 87,6 & 74,7 & 73,4 \\
\hline 19 & 76,5 & 78,5 & 76,4 & 78,6 & 86,8 & 74,2 & 73,1 \\
\hline
\end{tabular}

É possível verificar também que os valores medidos ficam acima do máximo estipulados pela NBR 10.151 (ABNT, 2000) para o conforto da população residencial, que é de $55 \mathrm{~dB}$ para períodos diurnos e $50 \mathrm{~dB}$ para períodos noturnos. Dessa forma, observando os valores medidos e os valores de predição, chegou-se à conclusão que a edificação se encontra na classe de ruído III.

\subsection{SIMULAÇÃO DO NÍVEL DE ISOLAMENTO SONORO}

Para o cálculo do índice Rw da fachada foi necessária a simulação dos diversos elementos constituintes dos sistemas de FVPo e FPoA. A simulação resultado em uma curva de isolamento sonoro (linha cheia) em $\mathrm{dBs}$, as frequências ordenadas em $1 / 3$ de oitava e a curva

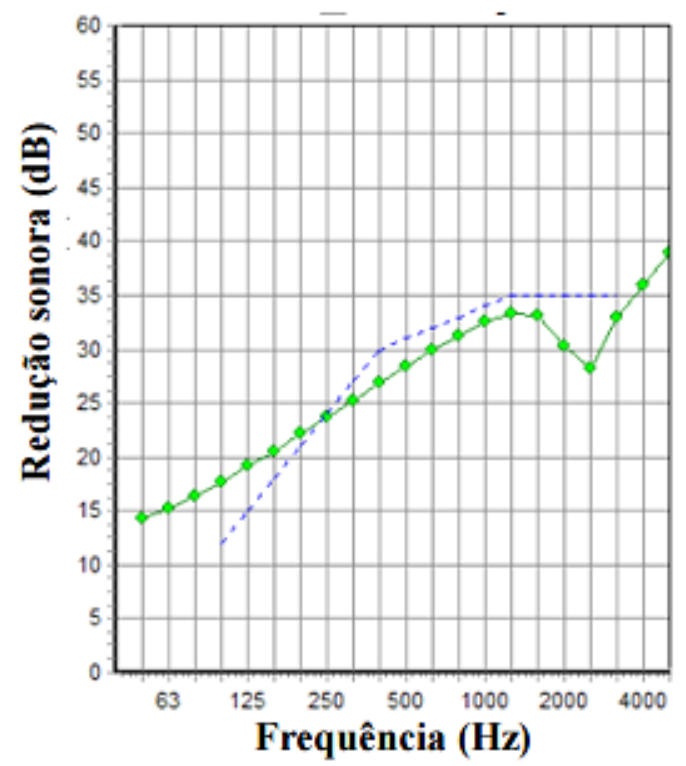

FIGURA 5 - Curva de isolamento sonora da esquadria. FONTE: Autoria própria. de referência (linha tracejada).

Primeiramente foi avaliada a esquadria da edificação que é comum ao cálculo de isolamento dos dois sistemas. Optou-se pelo vidro de $6 \mathrm{~mm}$ que resultou na seguinte curva de isolamento (Figura 5), frequência crítica de $2332 \mathrm{~Hz}$ e índice de redução sonora $(\mathrm{Rw})$ de $31 \mathrm{~dB}$.

A vedação do sistema de FPoA foi simulada da mesma forma, resultado em uma curva de isolamento sonora conforme a Figura 6, frequência crítica de $253 \mathrm{~Hz}$ e índice de redução sonora de $52 \mathrm{~dB}$.

Utilizando a Equação 2 calculou-se o RG do sistema de FPoA para os diferentes ambientes da edificação. $O$ resultado pode ser encontrado na Tabela 7.

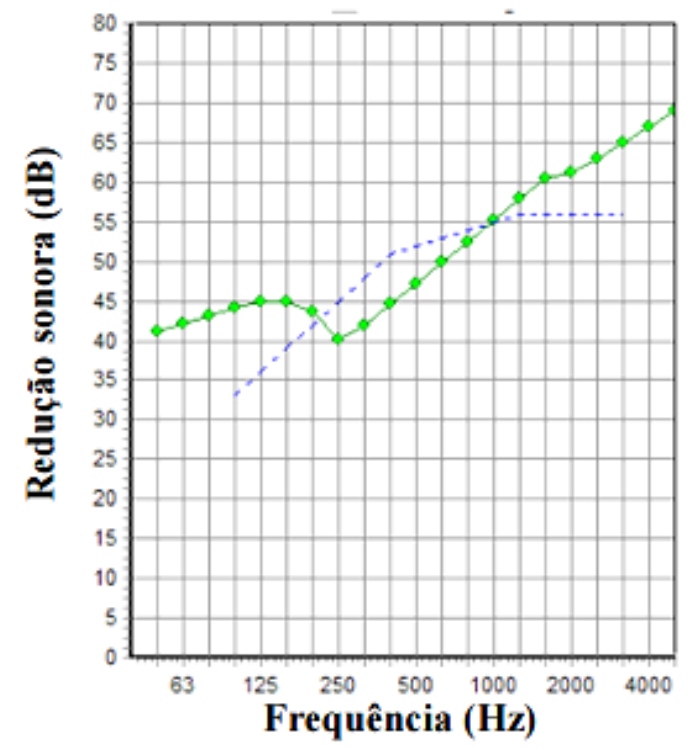

FIGURA 6 - Curva de isolamento sonoro da vedação do sistema de FPoA.

FONTE: Autoria própria. 
TABELA 7: Isolamento sonora do sistema de FPoA nos diferentes ambientes.

\begin{tabular}{c|c|c|c}
\hline Ambiente & Área da vedação $\left.\mathbf{( m}^{\mathbf{2}}\right)$ & Área Esquadria $\left(\mathbf{m}^{\mathbf{2}}\right)$ & RG (dB) \\
\hline Quarto & 6,71 & 1,80 & 38 \\
\hline Suíte & 6,77 & 2,40 & 37 \\
\hline Sala & 5,78 & 4,41 & 35 \\
\hline
\end{tabular}

FONTE: Autoria própria.

No caso do sistema de FVPo, foi calculado 0 isolamento sonoro do porcelanato com as juntas (Figura 7), do substrato (Figura 8) e por último o isolamento do sistema como um todo, levando em consideração as esquadrias. 0 resultado nos diferentes ambientes pode ser encontrado na Tabela 8.

A frequência crítica no porcelanato é de $1015 \mathrm{~Hz}$ e seu índice Rw é de $32 \mathrm{~dB}$. No substrato a frequência crítica é de $232 \mathrm{~Hz}$ e o isolamento sonoro $51 \mathrm{~dB}$.

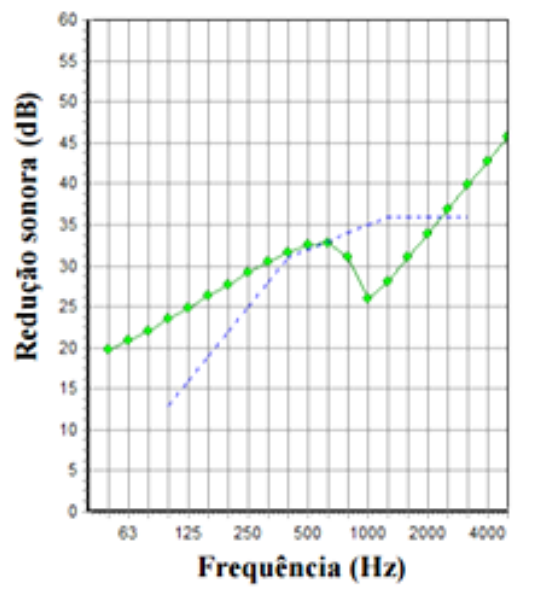

FIGURA 7 - Curva de isolamento sonoro do porcelanato no sistema de FVPo.

FONTE: Autoria própria.

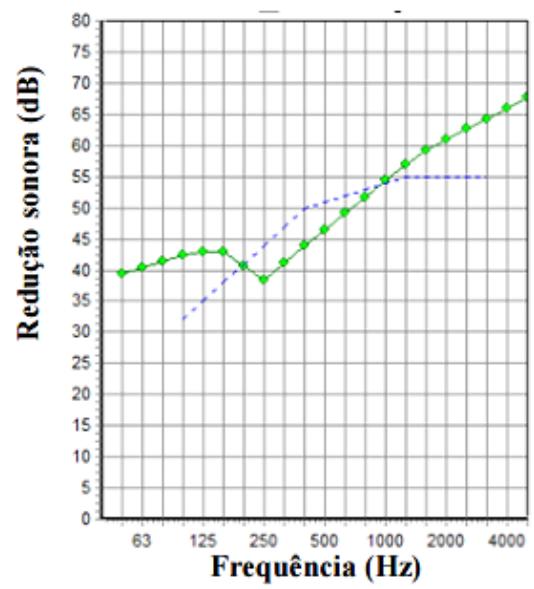

FIGURA 8 - Curva de isolamento sonoro do substrato no sistema de FVPo.

FONTE: Autoria própria.
Os resultados entre o isolamento sonoro dos sistemas foram semelhantes, diferindo um do outro por casas decimais, que quando arredondados para números inteiros acabaram ficando iguais. Apesar deste fato, o isolamento sonoro entre os dois sistemas possui mecanismos distintos. Enquanto o sistema de FPoA isola o ruído através de sua massa, o sistema de FVPo isola o ruído devido a diferença de impedância acústica entre seus materiais. Diferentes materiais possuem diferentes frequências críticas, favorecendo o isolamento sonoro em faixas de frequência distintas.

É possível concluir pelos resultados que a esquadria possui um papel muito importante no isolamento sonoro da fachada. Por ser o elemento com menor índice de isolamento sonoro da fachada, quanto maior for a sua área, maior será a diminuição do isolamento global desse sistema. Isto fica evidenciado quando se observam os resultados nas Tabelas 6 e 7.

Comparando os resultados das simulações com os requisitos sugeridos pela norma de desempenho, podemos chegar à conclusão que os dois sistemas atendem aos requisitos mínimos para a classe de ruído III.

Apesar do atendimento aos critérios da norma é necessário salientar que as frequências incidentes nesse estudo são em grande parte de baixa frequência (Figura 3). Quando analisados os gráficos de isolamento sonoro dos sistemas observa-se que os dois sistemas possuem grande isolamento sonoro em frequências altas e baixo isolamento em frequências baixas. Esse fato aliado ao baixo valor de frequência crítica dos elementos opacos pode trazer problemas de isolamento sonoro em baixas frequências. 
TABELA 8: Isolamento sonora do sistema de FVPo nos diferentes ambientes.

\begin{tabular}{c|c|c|c}
\hline Ambiente & Área da vedação $\left(\mathbf{m}^{\mathbf{2}}\right)$ & Área Esquadria $\left(\mathbf{m}^{\mathbf{2}}\right)$ & $\mathbf{R G}(\mathbf{d B})$ \\
\hline Quarto & 6,71 & 1,80 & 38 \\
\hline Suíte & 6,77 & 2,40 & 37 \\
\hline Sala & 5,78 & 4,41 & 35
\end{tabular}

No caso da FPoA, o sistema possui frequência crítica de $253 \mathrm{~Hz}$ enquanto o sistema de FVPo possui dois valores, a do porcelanato externo com valor de $1015 \mathrm{~Hz}$ e do substrato $232 \mathrm{~Hz}$. Essa diferença nos valores de frequência crítica do sistema de FVPo confere melhor isolamento sonoro em baixas frequências do que o sistema de FPoA. Uma forma de aprimorar o isolamento acústico do sistema de FVPo é utilizando isolantes acústicos no interior da camada ventilada, ou melhorar a própria esquadria da edificação.

\section{CONCLUSÕES}

No presente trabalho foram avaliados dois sistemas de fachadas (FVPo e FPoA), baseado nos requisitos e critérios definidos na NBR 15.575-4 (ABNT, 2013). Ambos os sistemas obtiveram resultados semelhantes, apesar de possuírem mecanismos de isolamento sonoro distintos. $\mathrm{O}$ sistema de FVPo, apesar de ter menor massa, possui um mecanismo de isolamento sonoro baseado nas diferenças de impedâncias acústica do revestimento e do substrato. Essa diferença de impedância leva ao um isolamento diferenciado das diversas faixas de frequência estudadas. A frequência crítica do porcelanato e do substrato também são diferentes diminuindo assim a perda de isolamento por ressonância.

O isolamento do sistema de FPoA é baseado em sua massa. Apesar de esperado um maior desempenho desse tipo de fachada, a concordância entra as frequências críticas dos materiais constituintes diminui o isolamento de todo sistema devido à perda de isolamento por ressonância.

Verifica-se também que esses dois sistemas possuem melhores índices de isolamento sonoro para altas frequências. Em projetos onde a solicitação acústica possui maiores valores de intensidade sonora para baixas frequências a FVPo pode proporcionar melhores índices de isolamento devido a diferenças de frequência crítica dos seus materiais.

Ainda foi possível analisar que as esquadrias das edificações são as grandes responsáveis por determinar o índice de isolamento sonoro global da fachada. Quanto maior a área da esquadria menor será o índice Rw da fachada.

Houve certa dificuldade na classificação da edificação quanto à classe de ruído em que ela se encontra. Essa dificuldade foi devido à escassez de normas nacionais sobre o assunto e a falta de valores limites para cada classe de ruído disposto na NBR 15.575-4 (ABNT, 2013). A metodologia de verificar o nível de ruído por equações de predição e medição in loco e comparar os resultados com a legislação existente, mostrou-se eficiente, mas não substitui a necessidade da inserção de parâmetros quantitativos na norma de desempenho.

$\mathrm{Na}$ medição in loco, verificou-se que o ruído produzido pelos veículos automotivos é maior que o permitido por norma e no caso de Distrito Federal, por lei. Esses valores de ruído de tráfego, produzidos acima do limite da norma, podem causar estresse, perturbação do ritmo biológico e aumento da pressão sanguínea dos frequentadores de ambientes adjacentes às vias estudadas. Para mitigar a emissão de ruídos nessas vias, faz-se necessário estudos específicos, mas é possível indicar algumas soluções, como por exemplo, a diminuição de velocidade máxima dos veículos, substituição do pavimento asfáltico comum por pavimento acústico, proibição de circulação de caminhões e veículos pesados, entre outros.

Este trabalho traz como principal contribuição a avaliação de desempenho acústico para sistemas inovadores no setor brasileiro de 
edificações.

Por fim, Brasília, por ser uma cidade com um desenho urbano singular, necessita de um estudo especifico a respeito da previsão dos níveis sonoros do ruído de tráfego, já que quando comparados os valores medidos com os valores previstos (nas equações de previsão existentes) verifica-se uma clara discrepância.

\section{AGRADECIMENTOS}

Os autores agradecem ao apoio de Conselho Nacional de Desenvolvimento Científico e Tecnológico - CNPq a este trabalho.

\section{REFERÊNCIAS BIBLIOGRÁFICAS}

ALCANTARA, L. C. G. Avaliação do conforto acústico de residências populares utilizando análise estatística de energia. Dissertação de Mestrado. Programa de Pósgraduação em Engenharia Mecânica, COPPE, Universidade Federal do Rio de Janeiro. Rio de Janeiro, 2010.

APARICIO-FERNÁNDEZ, C.; VIVANCOS, J. L.; FERRERGISBERT, P.; ROYO-PASTOR, R. Energy performance of a ventilated façade by simulation with experimental validation. Applied Thermal Engineering. v. 66, n.1-2,p. 563-570, 2014.

ASAKURA, T; MIAYJIMA, T; SAKAMOTO, S. Prediction method for sound from passing vehicle transmitted through building façade. Applied Acoustics. v. 74, n.5, p. 758-769, 2013.

ASSOCIAÇÃO BRASILEIRA DE NORMAS TÉCNICAS. NBR 10151 Acústica - Avaliação do ruído em áreas habitadas, visando o conforto da comunidade - Procedimentos. Rio de Janeiro, 2000.

.NBR 15575-1. Edificações Habitacionais Desempenho. Requisitos Gerais. Rio de Janeiro, 2013.

.NBR 15575-4. Edificações Habitacionais Desempenho. Sistemas de vedações verticais internas e externas - SVVIE. Rio de Janeiro, 2013.

BASTOS, P. K. X.; LEMONGE, A. C. C.; SIGILIANO, V. S.; RESENDE, S. C. Estudo do módulo de elasticidade de argamassa de revestimento sujeita à sucção de água pela base. 3o Congresso Português de Argamassas de construção, 2010.

CALIXTO, A. O ruído gerado pelo tráfego de veículos em "rodovias-grandes avenidas" situadas dentro do perímetro urbano de Curitiba, analisada sob parâmetros acústicos objetivos e seu impacto ambiental. Dissertação de Mestrado. Programa de Pós-graduação em Engenharia Mecânica, Universidade do Paraná. Curitiba, 2002.

CAMÂRA BRASILEIRA DA INDÚSTRIA DA CONSTRUÇÃO (CBIC). Desempenho de Edificações Habitacionais: Guia orientativo para atendimento à norma ABNT NBR 15575/2013. Brasília, 2013.

CAMPOS, K. F. Desenvolvimento de sistema de fixação de fachada ventilada com porcelanato de fina espessura. Dissertação de Mestrado. Programa de Pósgraduação em Engenharia Civil, Universidade Federal de Santa Catarina. Florianópolis, 2011.

CASTRO, T. M; TAVARES, C.R.G; LISOT, A.; KAMINATA, O.T. Caracterização de blocos cerâmicos acústicos produzidos com incorporação de lodo de lavanderia têxtil. Revista de engenharia sanitária e ambiental. v. 20, n.1, p. 47-54, 2015.

CHAKRABARTY, D.; SANTRA, S. C.; MUKHERJEE, A. Status of road traffic noise in Calcutta metropolis, India. Acoustic society of America. Journal of Acoustical Society of America. v.101, n.2, p. 125-129. 1997

CHIARELLO, J. A. Ventilação natural por efeito chaminé Estudo de modelo reduzido de pavilhões industriais. Dissertação de Mestrado. Programa de Pós-graduação em Engenharia Civil, Universidade Federal do Rio Grande do Sul. Porto Alegre, 2006.

CONSELHO REGIONAL DO MEIO AMBIENTE - CONAMA. "Resolução №002 de 8 de março de 1990 - Programa Nacional de Educação e Controle da Poluição Sonora". 1990.

CORBELLA, O.; YANAS, S. Em busca de uma arquitetura sustentável para os trópicos. Rio de Janeiro, Ed. Revan, 2003.

CUNHA, M. M. F. Desenvolvimento de um sistema construtivo para fachadas ventiladas. Dissertação de Mestrado. Instituto de Engenharia do Porto, Departamento de Engenharia Civil, Universidade do Minho. Porto, 2006.

DINTRANS, A.; PRÉNDEZ, M. A method of assessing measures to reduce road traffic noise: $A$ case study in Santiago, Chile. Applied Acoustics. v.74, n.12, p.14861491, 2013.

DISTRITO FEDERAL. “Lei no 4.092 de Janeiro de 2008 Controle da poluição sonora e limites máximos de intensidade de sons e emissão de ruídos resultantes de atividades urbana e rurais no Distrito Federal." Brasília, 2008. 
DUARTE, E. Estudo do isolamento acústico da paredes de vedação da moradia brasileira ao longo de sua história. Dissertação de Mestrado. Programa de Pós-graduação em Arquitetura, Universidade Federal de Santa Catarina. Florianópolis, 2005.

FERREIRA, J. A. C.; ZANNIN, P, H, T. Determinação de coeficientes de isolamento acústico: medições in situ e simulação computacional. Ambiente Construído. v. 7, n.2, p. 15-29, 2007.

GARAVELLI, S.L.; MORAES, A.C.; NASCIMENTO, J.R.R; NASCIMENTO, P.H.D.P.; MAROJA, A.M. Mapa de ruído como ferramenta de gestão da poluição sonora: estudo de caso de águas claras - DF. 40 Congresso LusoBrasileiro para o planejamento urbano regional, integrado e sustentável. Faro, Portugal. 2010.

GARCIA, A.; FAUS, L. J. Statistical analyis of noise levels in urban áreas. Applied Acoustics. v. 34, n.4, p.227-247, 1991

GARCIA, D. B.; VECCI, M. A. M.; RODRIGUES, F. C. Avaliação do isolamento sonoro aéreo de elementos de vedação na construção civil. Ambiente Construído. v. 8, n.1, p. 49-63, 2008.

GERRETSEN, E. Prediction of sound insulation in buildings: a tool to improve the acoustic quality. DAGA'03. Aechen, 2003.

HAMMAD, R. N. S.; GIBBS, B. M. The acoustic performance of building façade in hot climates: Part 1 Courtyards. Applied Acoustic. v.16, n.2, p.121-137, 1983.

HMSO. Departament of Transport (Dot) - Welsh Office. Calculation of Road Traffic Noise. London, 1988.

HENDRICKS, R.W. "Traffic Noise Attenuation as a Function of Ground and Vegetation". Techinical Report of California Departament of Transportation. Sacramento, California. 1995

IBRAM - Mapa de ruído de Brasília. Brasília, 2015.

INTERNACIONAL STANDARTS ORGANIZATION. ISO 717-1. Rating of sound insulation inbuildings and of building elements: airbornesound insulation. Geneva, Switzerland, 2013.

JUSTE, A. E. Estudo da resistência e da deformabilidade da alvenaria de bloco de concreto submetida a esforços de compressão. Dissertação de Mestrado. Programa de Engenharia Civil, Universidade de São Paulo. São Carlos, 2001.

KISS, P. Pulmões prediais. Revista Techne, edição 39, março, 1999.

KNUDSEN, V.O.; HARRIS, C.M. Acoutical Design in Architeture. Fifth printing, july, 1959.
LOPÉZ, P. F.; JENSEN, R. L.; HEISELBERG, P.; SANTIAGO, M. R. A. Experimental analisys and model validation of na opaque ventilated facade. Building and Environment. v. 56, n.10, p. 265-275, 2012.

LOPÉZ, P. F.; SANTIAGO, M. R. A. Sensitivity study of an opaque ventilated facade in the winter season in different climate zones in Spain. Renewable Energy. v. 75, n.3, p. 524-533, 2015.

MACIEL, A.C.F. Energia Incorporada de Fachadas Ventiladas nas Fases de Pré-uso, Uso e Manutenção. Estudo de Caso para Edificação Habitacional Modelo Típica do Plano Piloto de Brasília-DF. Dissertação de Mestrado. Programa de Pós-Graduação em Estruturas e Construção Civil, Universidade de Brasília. Brasília, 2013.

MACIEL, B.A.; RIBEIRO, R.J.C.; BIAS, E.S.; GARAVELLI, S.L.; CAVALCANTTI, M.M. Modelagem do ruído urbano como instrumento de gestão ambiental. Anais XIV Simpósio Brasileiro de Sensoriamento Remoto. Natal, Brasil. 2009.

MAKISHIMA, A.; MACKENZIE, J. D. Calculation of bulk modulus, shear modulus and Poisson's ratio of glass. Journal of Non-Crystalline Solids. v.17, n. 2, p.147-157, 1975.

MARDONES, M. D. M. Mapeamento dos níveis de ruído em Copacabana, Rio de Janeiro, através de simulação computacional. Dissertação de Mestrado. Programa de Pós-graduação em Engenharia Mecânica, COPPE, Universidade Federal do Rio de Janeiro. Rio de Janeiro, 2009

MARTINS, E. T. R. Caracterização de sistemas de reabilitação de fachadas: Soluções existentes e inovadoras. Dissertação de Mestrado. Programa de PósGraduação em Construção e Reabilitação Sustentáveis, Universidade do Minho. Portugal, 2013.

MULLER, A. Desenvolvimento de um Protótipo e Análise do Comportamento Térmico de Fachada Ventilada com Placas Cerâmicas de Grês Porcelanato. Tese de Doutorado. Programa de Pós-Graduação em Ciência e Engenharia de Materiais, Universidade Federal de Santa Catarina. Florianópolis, 2003.

NUNES, M. F. O. DORNELLES, G. T.; SOARES, I. N. Medidas de atenuação do ruído de tráfego urbano para o conforto acústico em áreas residenciais. Anais do V ENCAC. Fortaleza, 1999.

NUNES, M; RIBEIRO, H. Interferências do ruído do tráfego urbano na qualidade de vida: zona residencial de Brasília/DF. Cadernos metrópole. v. 19, n.1, p. 319-338. 2008.

ORGANIZAÇÃO MUNDIAL DA SAÚDE - Dísponível em < http://www.euro.who.int/en/health-

topics/environment-and-health/noise > - Acesso 02/12/2015 
PIMENTEL-SOUZA, F. Efeitos do ruído estressante. Anais da 49a Reunião Anual da SBPC, vol. 1. 2007

QUEIROZ, C. S. Avaliação do isolamento sonoro nas fachadas de edifícios residenciais. Estudo de caso: 0 processo evolutivo na avenida Beira Mar/Florianópolis. Dissertação de Mestrado, Programa de Pós-Graduação em Arquitetura e Urbanismo, Universidade Federal de Santa Catarina. Florianópolis, 2007.

SANJUAN, C.; SUÁREZ,M. J.; GONZÁLEZ, M.; PISTONO, J. BLANCO, E. Energy performance of an open-joint ventilated façade compared with a conventional sealed cavity façade. Solar Energy. v. 85, n.9, p. 1851-1863, 2011.

SANTOS FILHO, V. M. Análise de desempenho térmico e acústico de fachadas ventiladas de porcelanato à luz da norma de desempenho. Estudo de caso em Brasília - DF. Dissertação de Mestrado. Programa de Pós-Graduação em Estruturas e Construção Civil, Universidade de Brasília. Brasília, 2015.

SATTLER, M. A.; COSTA, A. C.; HANSEN, A. D. TERÁN, I. G. Avaliação de Impacto Em Ruído Ambiental Determinado Pela Introdução de Sistema Binário de Tráfego. Anais do I Simpósio Brasileiro de Metrologia em Acústica e Vibrações. Petrópolis, 1996. v. 1.

STAZI, F.; TOMASSONI, F.; VEGLIÒ, A.; DI PERNA, C. Experimental evaluation of ventilated walls with external clay cladding. Renewable Energy. v. 36, n. 12, p. 33733385, 2011.

STUBNA, I.; KOZÍK, T.; HANIC, F. Young's modulus and mechanical strength of porcelain at the firing in the cooling stage. Ceramics International. v. 18 , n. 15, p. 353354, 1992.

SUÁREZ, M. J.; SANJUAN, C.; GUTIÉRREZ, A. J.; PISTONO, J.; BLANCO, E. Energy evaluation of na horizontal open joint ventilated façade. Applied Thermal Engineering. Volume 37, n.5, p. 302-313, 2012.

WONG, P. C.; PRASAD, D.; BEHNIA, M. A new type of double-skin façade configuration for the hot humid climate. Energy and Buildings. v. 40, n. 10, p. 1941-1945, 2008. 